\title{
Relations of Job Stress, Burnout, Mindfulness and Job Satisfaction of Clinical Nurses
}

\author{
Jung $\operatorname{Im} \mathrm{Choi}^{1}$ and Myung Suk $\mathrm{Koh}^{2}$ \\ ${ }^{1}$ Sahmyook University, Graduate School, Nursing Department, Seoul, Korea \\ ${ }^{2}$ Sahmyook University, Nursing Department, Seoul, Korea \\ ${ }^{1}$ jwji51@naver.com, ${ }^{2}$ kohms@syu.ac.kr
}

\begin{abstract}
The study was conducted to determine the differences of the research variables according to general characteristics of clinical nurses and to understand the factors affecting the job satisfaction of clinical nurses in Korea. Subjects were 330 clinical nurses. The results are as shown below; job stress and burnout were different according to age groups, level of education, work units, current positions, and clinical careers. For mindfulness, differences were found according to age groups, level of education, and current positions. For job satisfaction, differences were found according to age groups, level of education, work unit, current positions

There was positive influence between job satisfaction and mindfulness, but both job stress and burnout were negative influences. The findings of this study implied that mindfulness had a positive impact, but job stress and burnout had a negative impact, on job satisfaction.
\end{abstract}

Keywords: Nurse, Job Stress, Job Satisfaction, Mindfulness, Burnout

\section{Introduction}

In order for hospitals to provide the best medical care, job satisfaction of clinical nurses must be carefully considered, since clinical nurses have the most interaction with patients. The quality of clinical nurses' work environment is believed to affect quality of patient care, to a greater extent than that of a positive working attitude. However, it has been reported that nurses tend to experience more work-related stress compared with other occupations, due to growing demand for quality as well as quantity in medical services, and due to the complexity of maintaining relationships with people of varying professions [1]. This stress generates negative job attitudes among nurses, and affects quality of medical service, which subsequently increases the rate of nursing errors, decreases patient satisfaction [2], and causes symptoms of burnout among nurses. Mindfulness in such circumstances lowers resistance to change in response to stressful situations, and facilitates cessation of dysfunctional behavior patterns [3]. In extant research nurse burnout has shown positive correlation with job stress and negative correlation with job satisfaction [4,5]. By contrast, mindfulness meditation is negatively correlated with job stress [1]. The purpose of this study is to investigate the effects of job stress, burnout, and mindfulness meditation on nurses' job satisfaction, and to obtain preliminary data regarding increasing job satisfaction of nurses.

\section{Methodology}

\subsection{Research Design and Subjects}

A descriptive design was used in the research, and subjects were clinical nurses of two general hospitals in Seoul who wanted to participate in this study. 


\subsection{Measurements}

To measure the interest in scientific learning, this study applied the Job Stress questionnaire[6], consisting of 30 questions using the five-step Likert scale. The Cronbach's $\alpha$ of this study was .921 . For measurement of burnout, we adapted the questionnaire developed by Pines, Aronson and Kafry[7],consisting of 20 questions and is also a five-step Likert scale. The Cronbach's $\alpha$ of this study was .962. For measurement of mindfulness, we adapted the questionnaire for mindfulness developed by Park[8], consisting of 20 questions using the fivestep Likert scale. The Cronbach's $\alpha$ of this study was .912. For measurement of job satisfaction we adapted the questionnaire for job satisfaction developed by Paula, Eugene, Dinah[9] consisting of 20 questions using the five-step Likert scale. The Cronbach's $\alpha$ of this study was .734 .

\subsection{Data Processing}

The SPSS WIN 20.0 program, using frequency, percentages, t-test, ANOVA, and Scheffe test for post hoc, was used for statistical processing ofdata collected in this study.

\section{Results}

\subsection{Characteristics of the Subjects}

The subjects were almost all women, 90.3\% (298); when the age ranges were checked, the $20^{\text {th }}$ age group was the largest group,54.5\%(180), and average age was $31.2 \pm 8.4$ years old. In education, diploma was the largest group, 57.3\%(189).Current positions were $79.1 \%(261)$ of staff nurses, average clinical experience was 5.9 \pm 3.4 years. $<$ Table 1>.

Table 1. General Characteristics of Participants

\begin{tabular}{|c|c|c|c|c|}
\hline Variables & Categories & $\mathrm{n}$ & $\%$ & $\mathrm{M} \pm \mathrm{SD}$ \\
\hline \multirow{2}{*}{ Gender } & Female & 298 & 90.3 & \\
\hline & Male & 32 & 9.7 & \\
\hline \multirow{4}{*}{ Age } & $20 \sim 29$ & 180 & 54.5 & \multirow{4}{*}{$31.2 \pm 8.4$} \\
\hline & $30 \sim 39$ & 84 & 25.5 & \\
\hline & $40 \sim 49$ & 47 & 14.2 & \\
\hline & $\geqq 50$ & 19 & 5.8 & \\
\hline \multirow{3}{*}{ Level of education } & Junior college & 189 & 57.5 & \\
\hline & University & 119 & 36.1 & \\
\hline & Graduate school or above & 21 & 6.4 & \\
\hline \multirow{3}{*}{ Work unit } & Inpatient & 196 & 59.7 & \\
\hline & Outpatient & 30 & 9.4 & \\
\hline & Special & 102 & 30.9 & \\
\hline \multirow{3}{*}{ Current position } & Staff nurse & 261 & 79.1 & \\
\hline & Charge nurse & 44 & 13.3 & \\
\hline & $\geq$ Head nurse & 25 & 7.6 & \\
\hline \multirow{3}{*}{$\begin{array}{c}\text { Clinical career } \\
\text { (years) }\end{array}$} & Less than 3 & 113 & 34.4 & \multirow{3}{*}{$5.9 \pm 3.4$} \\
\hline & More than 3 less than 5 & 74 & 22.6 & \\
\hline & More than 5 & 142 & 43.0 & \\
\hline
\end{tabular}

\subsection{Means of Job Stress, Burnout, Mindfulness and Job Satisfaction}

When we checked the means, firstly, mean of Job stress was $2.87 \pm 0.48$, burnout was $3.06 \pm 0.61$,mindfulness was $3.91 \pm 0.59$ and job satisfaction was $2.86 \pm 0.39<$ Table 2>. 
Table 2. Means of Research Variables

\begin{tabular}{|c|c|c|c|}
\hline Variables & Sub-dimensions & Mean & SD \\
\hline \multirow{7}{*}{ Job stress } & Total & 2.87 & 0.48 \\
\hline & Work load & 3.37 & 0.75 \\
\hline & Role conflict & 3.25 & 0.71 \\
\hline & Professional conflict & 2.93 & 0.71 \\
\hline & Conflict of staff & 2.44 & 0.62 \\
\hline & Conflict of senior & 2.46 & 0.69 \\
\hline & $\begin{array}{l}\text { Lack of knowledge } \\
\text { technology }\end{array}$ & 2.83 & 0.51 \\
\hline \multirow{4}{*}{ Burnout } & Total & 3.06 & 0.61 \\
\hline & Physical area & 3.51 & 0.71 \\
\hline & Emotional area & 2.96 & 0.67 \\
\hline & Mental area & 2.76 & 0.62 \\
\hline \multirow{5}{*}{ Mindfulness } & Total & 3.91 & 0.59 \\
\hline & Present awareness & 4.10 & 0.68 \\
\hline & Concentration & 3.93 & 0.69 \\
\hline & Non-judgement acceptance & 3.97 & 0.69 \\
\hline & De-centered attention & 3.65 & 0.80 \\
\hline \multirow{7}{*}{$\begin{array}{c}\text { Job } \\
\text { satisfaction }\end{array}$} & Total & 2.86 & 0.39 \\
\hline & Professional position & 3.06 & 0.52 \\
\hline & Pay & 1.88 & 0.81 \\
\hline & Work requirement & 2.98 & 0.51 \\
\hline & Autonomy & 3.20 & 0.81 \\
\hline & Administrative matters & 2.90 & 0.60 \\
\hline & Interactional relationship & 2.86 & 0.55 \\
\hline
\end{tabular}

\subsection{Differences of Research Variables According to the General Characteristics}

The differences of research variables according to the general characteristics were as follows.

First, in measurement of job stress, significant differences were observed between the $20^{\text {th }}$ and $40^{\text {th }}$ age groups $(\mathrm{F}=3.25, p=.022)$; in education level, there were significant differences in $\mathrm{BS}$, diploma, and MS degree ( $\mathrm{F}=3.08, p=.048)$, in order. Incomparison of the working units, there were significant differences among general wards, special unit, outpatient department, in order ( $\mathrm{F}=14.87, p<.001)$, and in positions, staff nurses were significantly higher than charge nurses $(\mathrm{F}=3.43$, $p=.033$ ).

For burnout, there were significant differences in the $20^{\text {th }}$ and $30^{\text {th }}$ age groups compared with the $40^{\text {th }}$ and $50^{\text {th }}$ age groups $(\mathrm{F}=17.90, p<.001)$. For education level, diploma and $\mathrm{BS}$ group were significantly higher than MS group ( $\mathrm{F}=9.97, p<.001)$. In comparison of working units, general ward nurses were significantly higher than special parts ( $\mathrm{F}=5.03, p=.007)$, in case of position, staff nurses were significantly higher than the charge and head nurses groups $(\mathrm{F}=14.64, p<.001)$, when 
comparing clinical experiences, the below 5 years group was significantly higher than the above 5 years group $(\mathrm{F}=10.26, p<.001)$.

Mindfulness according to general characteristics was as follows. First, men were significantly higher than women ( $\mathrm{t}=-.79, p=.006)$. There were significant differences among the age groups $(\mathrm{F}=3.6, p=.014)$, in education level, $\mathrm{BS}$ group was higher statistically than the diploma group $(\mathrm{F}=4.76, p=.009)$. In position, there were statistically significant differences among positions, but "post hoc could not be determined ( $\mathrm{F}=3.36, p=.036)$

For job satisfaction, there were significant differences between the $40^{\text {th }}, 50^{\text {th }}$ age group and $20^{\text {th }}(\mathrm{F}=9.24, p<.001)$. For education level, diploma and BS group were significantly lower than MS group $(\mathrm{F}=6.33, p=.002)$. In comparison of working units, general ward nurses were significantly lower than outpatient, special parts $(\mathrm{F}=6.62, p=.002)$, in case of position, charge nurses were significantly higher than staff nurse group $(\mathrm{F}=9.29, p<.001)$, ), when comparing clinical experiences, the below 3 years, and above 5 years group were significantly higher than the 3-5 years group $(\mathrm{F}=10.00, p<.001) .<$ Table $3>$.

\section{Table 3.Differences of Research Variables According to General Characteristics of Participants $(\mathrm{N}=330)$}

\begin{tabular}{|c|c|c|c|c|c|c|c|c|c|}
\hline \multirow{3}{*}{ Variables } & \multirow{3}{*}{ Items } & \multicolumn{2}{|c|}{ Job stress } & \multicolumn{2}{|c|}{ Burnout } & \multicolumn{2}{|c|}{ Mindfulness } & \multicolumn{2}{|c|}{$\begin{array}{c}\text { Job } \\
\text { Satisfaction }\end{array}$} \\
\hline & & \multirow{2}{*}{$\begin{array}{l}\text { Mean } \\
(\mathrm{SD})\end{array}$} & $\mathrm{t} / \mathrm{F}(p)$ & \multirow{2}{*}{$\begin{array}{l}\text { Mean } \\
(\mathrm{SD})\end{array}$} & $\mathrm{t} / \mathrm{F}(p)$ & \multirow{2}{*}{$\begin{array}{l}\text { Mean } \\
(\mathrm{SD})\end{array}$} & $\mathrm{t} / \mathrm{F}(p)$ & \multirow{2}{*}{$\begin{array}{l}\text { Mean } \\
\text { (SD) }\end{array}$} & $\mathrm{t} / \mathrm{F}(p)$ \\
\hline & & & Scheffe & & Scheffe & & Scheffe & & Scheffe \\
\hline \multirow[t]{2}{*}{ Gender } & Female & $\begin{array}{l}2.88 \\
(0.48)\end{array}$ & \multirow{2}{*}{$\begin{array}{l}.56 \\
(.811)\end{array}$} & $\begin{array}{l}3.07 \\
(0.61)\end{array}$ & \multirow{2}{*}{$\begin{array}{l}.83 \\
(.545)\end{array}$} & $\begin{array}{l}3.90 \\
(0.56)\end{array}$ & \multirow{2}{*}{$\begin{array}{l}-.79 \\
(.006)\end{array}$} & $\begin{array}{l}2.86 \\
(0.39)\end{array}$ & \multirow{2}{*}{$\begin{array}{c}-.96 \\
(.716)\end{array}$} \\
\hline & Male & $\begin{array}{l}2.83 \\
(0.52)\end{array}$ & & $\begin{array}{l}2.97 \\
(0.59)\end{array}$ & & $\begin{array}{l}4.10 \\
(0.79)\end{array}$ & & $\begin{array}{l}2.93 \\
(0.38)\end{array}$ & \\
\hline \multirow[t]{4}{*}{ Age(yr) } & a. $20 \sim 29$ & $\begin{array}{l}2.92 \\
(0.47)\end{array}$ & \multirow{4}{*}{$\begin{array}{l}3.25 \\
(.022) \\
a>c\end{array}$} & $\begin{array}{l}3.20 \\
(0.50)\end{array}$ & & $\begin{array}{l}3.88 \\
(0.58)\end{array}$ & \multirow{4}{*}{$\begin{array}{l}3.58 \\
(.014)\end{array}$} & $\begin{array}{c}2.78 \\
(0.36)\end{array}$ & \multirow{4}{*}{$\begin{array}{c}9.24 \\
(<.001) \\
a<c, d\end{array}$} \\
\hline & b. $30 \sim 39$ & $\begin{array}{l}2.87 \\
(0.50)\end{array}$ & & $\begin{array}{l}3.09 \\
(0.64)\end{array}$ & $\begin{array}{l}17.90 \\
(<001)\end{array}$ & $\begin{array}{l}3.84 \\
(0.58)\end{array}$ & & $\begin{array}{c}2.87 \\
(0.33)\end{array}$ & \\
\hline & c. $40 \sim 49$ & $\begin{array}{l}2.69 \\
(0.50)\end{array}$ & & $\begin{array}{l}2.68 \\
(0.69)\end{array}$ & a,b $>$ c,d & $\begin{array}{l}4.05 \\
(0.66)\end{array}$ & & $\begin{array}{c}3.05 \\
(0.43)\end{array}$ & \\
\hline & d. $\geqq 50$ & $\begin{array}{l}2.75 \\
(0.43)\end{array}$ & & $\begin{array}{l}2.45 \\
(0.52)\end{array}$ & & $\begin{array}{l}4.25 \\
(0.40)\end{array}$ & & $\begin{array}{c}3.12 \\
(0.54)\end{array}$ & \\
\hline \multirow{3}{*}{$\begin{array}{l}\text { Level of } \\
\text { Education }\end{array}$} & $\begin{array}{l}\text { a.Junior } \\
\text { college }\end{array}$ & $\begin{array}{l}2.85 \\
(0.45)\end{array}$ & \multirow{3}{*}{$\begin{array}{l}3.07 \\
(.048)\end{array}$} & $\begin{array}{l}3.13 \\
(0.54)\end{array}$ & \multirow{3}{*}{$\begin{array}{l}9.97 \\
(<.001) \\
a, b>c\end{array}$} & $\begin{array}{l}3.83 \\
(0.55)\end{array}$ & \multirow{3}{*}{$\begin{array}{l}4.76 \\
(.009) \\
a<b\end{array}$} & $\begin{array}{c}2.83 \\
(0.36)\end{array}$ & \multirow{3}{*}{$\begin{array}{c}6.33 \\
(.225) \\
\mathrm{a}, \mathrm{b}<\mathrm{c}\end{array}$} \\
\hline & $\begin{array}{l}\text { b. } \\
\text { University }\end{array}$ & $\begin{array}{l}2.9 \\
3(0.52)\end{array}$ & & $\begin{array}{l}3.03 \\
(0.66)\end{array}$ & & $\begin{array}{l}4.01 \\
(0.65)\end{array}$ & & $\begin{array}{c}2.88 \\
(0.38)\end{array}$ & \\
\hline & $\begin{array}{l}\text { c..Above } \\
\text { graduate } \\
\text { School }\end{array}$ & $\begin{array}{l}2.66 \\
(0.57)\end{array}$ & & $\begin{array}{l}2.52 \\
(0.70)\end{array}$ & & $\begin{array}{l}4.09 \\
(0.54)\end{array}$ & & $\begin{array}{c}3.14 \\
(0.54)\end{array}$ & \\
\hline \multirow{3}{*}{$\begin{array}{l}\text { Work } \\
\text { unit }\end{array}$} & a. Inpatient & $\begin{array}{l}2.97 \\
(0.44)\end{array}$ & \multirow{3}{*}{$\begin{array}{l}14.87 \\
(<.001) \\
a>b>c\end{array}$} & $\begin{array}{l}3.14 \\
(0.60)\end{array}$ & & $\begin{array}{l}3.88 \\
(0.59)\end{array}$ & \multirow{3}{*}{$\begin{array}{l}.53 \\
(.587)\end{array}$} & $\begin{array}{l}2.80 \\
(0.36)\end{array}$ & \multirow{3}{*}{$\begin{array}{c}6.62 \\
(.002) \\
a<b, c\end{array}$} \\
\hline & $\begin{array}{l}\text { b. } \\
\text { Outpatient }\end{array}$ & $\begin{array}{l}2.52 \\
(0.41)\end{array}$ & & $\begin{array}{l}2.84 \\
(0.69)\end{array}$ & $\begin{array}{l}5.03 \\
(.007)\end{array}$ & $\begin{array}{l}3.96 \\
(0.69)\end{array}$ & & $\begin{array}{l}3.02 \\
(0.37)\end{array}$ & \\
\hline & c. Special & $\begin{array}{l}2.77 \\
(0.53)\end{array}$ & & $\begin{array}{l}2.97 \\
(0.59)\end{array}$ & $a>b$ & $\begin{array}{l}3.95 \\
(0.57)\end{array}$ & & $\begin{array}{l}2.94 \\
(0.42)\end{array}$ & \\
\hline $\begin{array}{l}\text { Current } \\
\text { position }\end{array}$ & $\begin{array}{ll}\text { a. } & \text { Staff } \\
\text { nurse } & \end{array}$ & $\begin{array}{l}2.90 \\
(0.49)\end{array}$ & $\begin{array}{l}3.43 \\
(.033)\end{array}$ & $\begin{array}{l}3.15 \\
(0.56)\end{array}$ & $\begin{array}{l}14.64 \\
(<.001)\end{array}$ & $\begin{array}{l}3.87 \\
(0.59)\end{array}$ & $\begin{array}{l}3.36 \\
(.036)\end{array}$ & $\begin{array}{c}2.82 \\
(0.36)\end{array}$ & \\
\hline
\end{tabular}




\begin{tabular}{|c|c|c|c|c|c|c|c|c|c|}
\hline & $\begin{array}{l}\text { b.Charge } \\
\text { nurse }\end{array}$ & $\begin{array}{l}2.70 \\
(0.42)\end{array}$ & $a>b$ & $\begin{array}{l}2.67 \\
(0.67)\end{array}$ & $a>b, c$ & $\begin{array}{l}4.10 \\
(0.53)\end{array}$ & & $\begin{array}{c}3.08 \\
(0.42)\end{array}$ & $\begin{array}{c}9.29 \\
(<.001)\end{array}$ \\
\hline & $\begin{array}{l}\text { c. } \geq \text { Head } \\
\text { nurse }\end{array}$ & $\begin{array}{l}2.83 \\
(0.47)\end{array}$ & & $\begin{array}{l}2.80 \\
(0.68)\end{array}$ & & $\begin{array}{l}4.02 \\
(0.62)\end{array}$ & & $\begin{array}{c}2.95 \\
(0.45)\end{array}$ & $\mathrm{a}<\mathrm{b}$ \\
\hline \multirow[t]{3}{*}{$\begin{array}{l}\text { Clinical } \\
\text { career(yr) }\end{array}$} & a. $\angle 3$ & $\begin{array}{l}2.91 \\
(0.48)\end{array}$ & 3.70 & $\begin{array}{l}3.11 \\
(0.55)\end{array}$ & 10.26 & $\begin{array}{l}3.87 \\
(0.59)\end{array}$ & 1.76 & $\begin{array}{c}2.86 \\
(0.35)\end{array}$ & \multirow{3}{*}{$\begin{array}{c}10.00 \\
(<.001) \\
\text { a,c }>\text { b }\end{array}$} \\
\hline & b. $3 \leq-\angle 5$ & $\begin{array}{l}2.95 \\
(0.49)\end{array}$ & \multirow{2}{*}{$(.026)$} & $\begin{array}{l}3.27 \\
(0.53)\end{array}$ & $(<.001)$ & $\begin{array}{l}3.84 \\
(0.55)\end{array}$ & \multirow{2}{*}{$(.174)$} & $\begin{array}{c}2.71 \\
(0.34)\end{array}$ & \\
\hline & c. $\geq 5$ & $\begin{array}{l}2.78 \\
(0.47)\end{array}$ & & $\begin{array}{l}2.90 \\
(0.66)\end{array}$ & $\mathrm{a}, \mathrm{b}>\mathrm{c}$ & $\begin{array}{l}3.98 \\
(0.61)\end{array}$ & & $\begin{array}{c}2.95 \\
(0.41)\end{array}$ & \\
\hline
\end{tabular}

\subsection{Correlations among the Variables}

The correlations of variables were as follows:job stress with burnout was $\mathrm{r}=.576(p<.001)$, job stress with mindfulness was $\mathrm{r}=-.279(p<.001)$, job stress with job satisfaction was $\mathrm{r}=-$ $.530(p<.001)$, burnout with mindfulness was $(\mathrm{r}=-.415(p<.001)$, burnout with job satisfaction was $\mathrm{r}=-.669(p<.001)$, and job satisfaction with mindfulness was $\mathrm{r}=.171(p=.002)<$ Table $4>$.

Table 4.Correlations among the Variables

\begin{tabular}{lccc}
\hline & $\begin{array}{c}\text { Job stress } \\
\mathrm{r}(p)\end{array}$ & $\begin{array}{c}\text { Burnout } \\
\mathrm{r}(p)\end{array}$ & $\begin{array}{c}\text { Mindfulness } \\
\mathrm{r}(p)\end{array}$ \\
\hline Job stress & 1 & & \\
Burnout & $.576(<.001)$ & 1 & \\
Mindfulness & $-.279(<.001)$ & $-.415(<.001)$ & 1 \\
Job satisfaction & $-.530(<.001)$ & $-.669(<.001)$ & $.171(.002)$ \\
\hline
\end{tabular}

\subsection{Factors Predicting Job Satisfaction}

In the results of hierarchical regressions, job satisfaction was most influenced by burnout, followed by job stress and mindfulness. The three variables, that is, burnout, job stress, and mindfulness explained 52.0 percent. $\langle$ Table 5>.

Table 5. Factors Predicting Job Satisfaction

\begin{tabular}{|c|c|c|c|c|c|c|c|c|}
\hline \multirow{2}{*}{ Model } & \multicolumn{2}{|c|}{ Model 1} & \multicolumn{2}{|c|}{ Model 2} & \multicolumn{2}{|c|}{ Model 3} & \multicolumn{2}{|c|}{ Model 4} \\
\hline & $\beta$ & $\mathrm{t}(p)$ & $\beta$ & $\mathrm{t}(p)$ & $\beta$ & $\mathrm{t}(p)$ & $\beta$ & $\mathrm{t}(p)$ \\
\hline \multicolumn{9}{|l|}{ Constant } \\
\hline Age & .39 & $\begin{array}{r}4.52 \\
(<.001)\end{array}$ & .30 & $\begin{array}{r}3.89 \\
(<.001)\end{array}$ & .12 & $\begin{array}{r}1.76 \\
(.079)\end{array}$ & .13 & $\begin{array}{r}1.80 \\
(.072)\end{array}$ \\
\hline $\begin{array}{l}\text { Level of education } \\
\text { University }\end{array}$ & .03 & $\begin{array}{r}-.458 \\
(.647)\end{array}$ & .04 & $\begin{array}{r}.89 \\
(.375)\end{array}$ & .01 & $\begin{array}{r}.22 \\
(.830)\end{array}$ & .03 & $\begin{array}{r}.67 \\
(.503)\end{array}$ \\
\hline Gradurteschoolabove & . 10 & $\begin{array}{r}1.55 \\
(.121)\end{array}$ & .08 & $\begin{array}{r}1.43 \\
(.151)\end{array}$ & .03 & $\begin{array}{r}.69 \\
(.493)\end{array}$ & .03 & $\begin{array}{r}.71 \\
(.477)\end{array}$ \\
\hline $\begin{array}{l}\text { Work unit } \\
\text { Outpatient unit }\end{array}$ & . 10 & $\begin{array}{r}1.82 \\
(.069)\end{array}$ & .01 & $\begin{array}{r}-.27 \\
(.791)\end{array}$ & .013 & $\begin{array}{r}.30 \\
(.761)\end{array}$ & .01 & $\begin{array}{r}.13 \\
(.893)\end{array}$ \\
\hline Special unit & .10 & $\begin{array}{r}1.73 \\
(.077)\end{array}$ & .03 & $\begin{array}{r}.57 \\
(.571)\end{array}$ & .04 & $\begin{array}{r}.86 \\
(.390)\end{array}$ & .03 & $\begin{array}{r}.82 \\
(.413)\end{array}$ \\
\hline $\begin{array}{l}\text { Position } \\
\text { Charge nurse }\end{array}$ & .02 & $\begin{array}{r}0.32 \\
(.752)\end{array}$ & .01 & $\begin{array}{r}.21 \\
(.834)\end{array}$ & -.01 & $\begin{array}{r}-.16 \\
(.876)\end{array}$ & -.01 & $\begin{array}{r}-.10 \\
(.922)\end{array}$ \\
\hline Head nurse & $.18^{-}$ & $\begin{array}{l}-2.48 \\
(.014)\end{array}$ & .13 & $\begin{array}{l}-1.96 \\
(.051)\end{array}$ & -.08 & $\begin{array}{r}1.45 \\
(.148)\end{array}$ & -.08 & $\begin{array}{l}-1.41 \\
(.157)\end{array}$ \\
\hline
\end{tabular}




\begin{tabular}{|c|c|c|c|c|c|c|c|c|}
\hline $\begin{array}{l}\text { Career } \\
\text { More than } 3 \text { less than } 5\end{array}$ & .21 & $\begin{array}{r}-3.61 \\
(<.001)\end{array}$ & $\begin{array}{r}- \\
.18\end{array}$ & $\begin{array}{r}-3.44 \\
(.001)\end{array}$ & -.12 & $\begin{array}{r}-2.51 \\
(0.12)\end{array}$ & -.11 & $\begin{array}{l}-2.46 \\
(.014)\end{array}$ \\
\hline More than 5 & .15 & $\begin{array}{l}-1.97 \\
(.049)\end{array}$ & $\begin{array}{r}- \\
.14\end{array}$ & $\begin{array}{l}-2.01 \\
(.044)\end{array}$ & -.07 & $\begin{array}{l}-1.07 \\
(.285)\end{array}$ & -.07 & $\begin{array}{l}-1.12 \\
(.263)\end{array}$ \\
\hline Job stress & & & $\begin{array}{r}- \\
.47\end{array}$ & $\begin{array}{r}-9.83 \\
(<.001)\end{array}$ & -.21 & $\begin{array}{r}-4.12 \\
(<.001)\end{array}$ & -.23 & $\begin{array}{r}-4.45 \\
(<.001) \\
\end{array}$ \\
\hline Burnout & & & & & -.50 & $\begin{array}{r}-9.27 \\
(<.001)\end{array}$ & -.55 & $\begin{array}{r}-9.98 \\
(<.001)\end{array}$ \\
\hline Mindfulness & & & & & & & .15 & $\begin{array}{c}3.32 \\
(.001)\end{array}$ \\
\hline $\mathrm{R}^{2}\left(\operatorname{Adj} \mathrm{R}^{2}\right)$ & & $74(.150)$ & & $68(.348)$ & & $03(.486)$ & & $20(.502)$ \\
\hline $\mathrm{F}(p)$ & & $2(<.001)$ & 18.3 & $6(<.001)$ & 29.0 & $1(<.001)$ & 28.3 & $6(<.001)$ \\
\hline
\end{tabular}

\section{Discussion}

The average job stress score of nurses participating in this study was 2.87 , less than the average of 3.33-3.37 reported by Han et al.[10]. We consider this difference to be the result of variation between participant groups and variation in the days on which measurements were made; these factors require investigation by the nursing organizations. Regarding burnout, in our study the average score was 3.06, greater than the score of 2.98 obtained from measurements of nursing professionals conducted by Insurance Review Nurses [11]. A wide range of factors is likely responsible for this variation between results; one may be varying levels of job satisfaction between advanced practice nurses, who perform professional nursing practice, and general practice nurses, who provide nursing care to diverse and complex cases. Studies comparing these two groups of nurses are therefore needed to confirm these results. By contrast, the average mindfulness meditation score obtained in this study was 3.91, which is less than the average score of 4.09 reported elsewhere [1]. Mindfulness meditation immediately decreases negative emotional response and increases cognitive assessment scores; these effects facilitate the cessation of dysfunctional behavior patterns in stressful situations [12]. Current research therefore stresses the importance of mindfulness training programs to increasing nurses' job satisfaction.

Regarding differences in job stress according to general characteristics, we found significant differences depending on age, educational level, department, position, and clinical experience. Regarding age, nurses in their twenties had significantly higher job stress scores than those in their forties. This result may suggest that nurses in their twenties experience higher workload. However, this contrasts with a study by Han et al. [10], who found that job stress increases with increasing age. This finding may be due to differences in the characteristics of jobs performed by different age groups; nonetheless, this issue requires further investigation. Regarding burnout scores according to general characteristics, statistically significant differences were found depending on age, educational level, department, position, and clinical experience. That is, general nurses in their twenties and thirties, those with a vocational degree, those working in general wards, compared to those with more than 5 years of experience had higher burnout scores. These results are similar to those of Kwon \& Lee, who used the same instrument [4]. Therefore, programs and research should be implemented and conducted to reduce clinical nurses' burnout rates. In particular, nurses with less experience and of lower position experience greater loss of clinical problemsolving ability, and engage in more trial-and-error behavior, which promotes burnout. Thus, development of programs to prevent burnout resulting from work experience or position is vital. Conversely, nurses who were male, in their fifties, had a bachelor's degree, head nurses, and nurses with five or more years of working experience had greater mindfulness scores; this result was similar to that of $\mathrm{Oh} \&$ Koh [1]. However, since few studies have examined mindfulness in clinical nurses, this topic should be the subject of detailed future investigation 
Finally, job satisfaction was most influenced by burnout, followed by job stress and mindfulness. The three variables (burnout, job stress, and mindfulness) explained 52.0 percent. The findings of this study suggested that mindfulness had a positive impact, but job stress and burnout had a negative impact,on job satisfaction. Based on these findings, various types of solutions to increasing job satisfaction of clinical nurses should be developed and administered. It also suggested that consistent and various further studies on mindfulness as a solution should be conducted.

\section{Acknowledgements}

This article is a revision of the first author's master's thesis from Sahmyook University

\section{References}

[1] J. K. Oh and H. S.Koh, "The Mediating Effect of Mindfulness in the Relationship between Emotional Intelligence and Stress among Clinical Nurses",The Korean Journal of Stress Research, vol. 22, no. 3, (2014), pp. 139-147.

[2] C. Garrett, "The effect of nurse staffing patterns on medical errors and nurse burnout", Association of Operating Room Nurse Journal, vol. 87, (2008), pp. 1191-1204.

[3] J. A. Hayes, "What are the benefits of mindnfulness?",A practice review of psychotherapy-related research. Psychotherapy: Theory, Research and Practice, vol. 48, no. 2, (2011), pp. 198-208.

[4] K. J. Kwon and S. H. Lee, "Occupational Stress and Coping Styles as Factors Affecting the Burnout of Clinical Nurses", Journal of Korean Academy of Nursing Administration, vol. 18, no. 4, (2012), pp. 383-393.

[5] D. S. Byun and Y. H.Yom, "Factors affecting te burnout of clinical nurses-focused on emotional labor",Journal of Korean Academy of Nursing Administration, vol. 15, no. 3,(2009), pp. 444-454.

[6] M. J.Kim and M. O.Gu, "The development of the stress measurement tool for staff nurses working in the hospital", Journal of Korean Academy of Nursing, vol. 14, no. 2, (1984), pp. 28-37.

[7] A. M.Pines,E Kafry and D.Aronson.Burnout, "From tedium to personal growth. Free press New York",MacMillian Publishing Co.,(1981).

[8] S. H. Park, "Development of the mindfulness scale", Dissertation, Catholic University,(2006).

[9] L. S.Paula,B. P.Eugene, B. S.Dinah and M. H.Ann, "Measurement of levels of satisfaction of nurses within their professional work situation”, Nursing Research, vol. 26, no. 2,(1978), pp. 114-120.

[10] A. K.Han,O. S.Kim and J. S.Won, "A Study on Job Stress and Coping Method by the Personality Types of Clinical Nurses", Journal of Korean Clinical Nursing Research, vol. 13, no. 2,(2007), pp. 125-136.

[11] S. I.Jeong, E. N.Lee and Y. S.Song, "The Main \& Buffering Effects of Perceived Social Support on Burnout of Insurance Review Nurses", Journal of Korean Academy of Nursing Administration, vol. 12, no. 3,(2006), pp. 482-490.

[12] C. S.Mackenzie, P. A.Poulin and R S. Carlson, "A brief mindfulness-based stress reduction intervention for nurses and nurse aides", Applied Nursing Research, vol. 19, (2006), pp. 105-109.

\section{Authors}

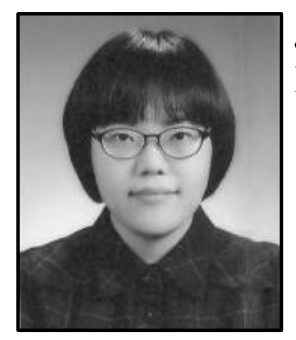

Jung Im Choi

RN, Sahmyook Medical Center,Seoul,Korea 
International Journal of Bio-Science and Bio-Technology

Vol.7, No.3 (2015)

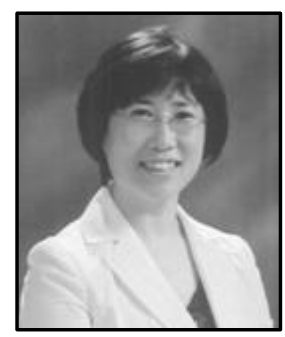

Myung Suk Koh

Professor, Department of Nursing, Sahmyook University, Seoul, Korea 